\title{
A microarray analysis of full depth knee cartilage of ovariectomized rats
}

\author{
Anne C Bay-Jensen ${ }^{1 *}$, Rasmus H Nielsen ${ }^{1}$, Toni Segovia-Silvestre', Moïse Azria ${ }^{2}$, Frank Staedtler ${ }^{2}$, Martin Letzkus ${ }^{2}$, \\ Nicole Hartmann ${ }^{2}$, Arndt $\mathrm{H} \mathrm{Brachat}^{2}$ and Morten A Karsdal ${ }^{1}$
}

\begin{abstract}
Background: This short communication focuses the on articular cartilage and the subchondral bone, both of which play important roles in the development of osteoarthritis $(\mathrm{OA})$. There are indications that estrogendeficiency, as the post-menopausal state, accelerate the development of OA.

Findings: We investigated, which extracellular matrix (ECM) protein, proteases and different pro-inflammatory factors was up- or down-regulated in the knee joint tissue in response to estrogen-deficiency in rats induced by ovariectomy. These data support previous findings that several metalloproteinases (MMPs) and cysteine proteases are co-regulated with numerous collagens and proteoglycans that are important for cartilage integrity. Furthermore quite a few pro-inflammatory cytokines were regulated by estrogen deprivation.

Conclusion: We found multiple genes where regulated in the joint by estrogen-deficiency, many of which correspond well with our current knowledge of the pathogenesis of OA. It supports that estrogen-deficiency (e.g. OVX) may accelerate joint deterioration. However, there are also data that draw attention the need for better understanding of the synergy between proteases and tissue turnover.
\end{abstract}

\section{Introduction}

Post-menopausal women have a higher incident rate of osteoarthritis (OA) and osteoporosis (OP) than that of age-matched men $[1,2]$. There are indications that perimenopausal women receiving estrogen replacement therapy (ERT) had lower risk of developing radiographic knee and hip OA and that the protective effect was increased with increased duration of ERT treatment [1]. This is in line with findings that also bone turnover is increased due to estrogen-deficiency [3]. Mouritzen et al. (2005) found that urinary levels of cartilage and bone turnover markers (i.e. C-terminal telopeptide of type I and II collagen, CTX-I/II) were increased in post-menopausal women compared to pre-menopausal women. Since then it has been shown that selective estrogenreceptor modulators (SERMs) given to postmenopausal women decrease the level of CTX-II about $50 \%$ of baseline (12-month follow-up). These markers are generated by enzymatic processing of type II and I collagen, respectively, which is a result of induced cellular

\footnotetext{
* Correspondence: acbj@nordicbioscience.com

'Cartilage biology and biomarkers, Nordic Bioscience, Herlev, Denmark Full list of author information is available at the end of the article
}

responses. Furthermore, it has been shown that adult articular cartilage expresses estrogen receptors and these can be activated and thereby induce production of extracellular matrix (ECM) molecules (e.g. proteoglycans and collagens) [4]. Ovariectomy of rats led to similar results; estrogen-deficiency led to cartilage damage and increased bone resorption, which could be prevented by administrations of estrogen [57]. Comparable studies have been done in other species [8]. We investigated which genes related to cartilage turnover and integrity was regulated in response to estrogen deprivation by comparing the mRNA expression between sham and ovariectomized rats.

\section{Methods}

Six month old female rats were either ovariectomized (OVX, $\mathrm{n}=5)$ or sham operated $(\mathrm{n}=5)$. After recovery the rats were house under standard conditions for 8 weeks, and terminated. All animal experiments were approved by the local ethical authorities ("Dyreforsøgstilsynet", approval no. DK-2006/561-1239). The knees were isolated right after termination and the cartilage and subchondral compartment was detached from the
C Biomed Central

(c) 2010 Bay-Jensen et al; licensee BioMed Central Ltd. This is an Open Access article distributed under the terms of the Creative Commons Attribution License (http://creativecommons.org/licenses/by/2.0), which permits unrestricted use, distribution, and reproduction in any medium, provided the original work is properly cited. 
tibia and flash frozen in liquid nitrogen (Figure 1). Total RNA was isolated from the pulverized tissue using standard phenol based extraction method. The mRNA was hybridized to an Affymetrix GeneChip [9].

Expression data was retrieved from the internal microarray database discriminating 1.5 -fold and 2-fold significant ( $\mathrm{p}<0.05$ by Student's t-test) increased or decreased expression of individual genes when comparing the OVX and sham group, which gave a list of 2.959 and 579 genes, respectively. An objective search where made on database terms: Extracellular matrix protein, Protease/Proteinase, Cytokine, Growth factor, Bone and Cartilage. From these searched, literature based and subjectively guided sorting was done on structural proteins involved in cartilage and bone tissue remodeling and destruction, as well as cartilage and bone related factors. Each of the genes in question was reviewed by searches (term: "gene name" cartilage and/or bone) on Pubmed http://www.ncbi.nlm.nih.gov/pubmed/, IHOP http:// www.ihop-net.org/UniPub/iHOP/, Rat genome database http://rgd.mcw.edu/tools/genes/ and HUGO gene nomenclature Committee http://www.genenames.org/.

\section{Expression of ECM proteins in response to estrogen deficiency}

A total of nine relevant ECM proteins genes were found to be differentially expressed in the OVX group compared to the sham group (Figure 2A). Only one ECM gene were significantly up-regulated; Dentin matrix protein 1 (DMP1). DMP1 is expressed in mineralized tissues such as hypertrophic cartilage and bone, where it can bind $\mathrm{Ca}^{2+}$ and regulate matrix mineralization [10]. There were eight ECM genes down-regulated. Fibromodulin (FMOD) is known to interact with both type I and II collagen in bone and cartilage, respectively [11]. Through its binding of collagens it aids stabilization of

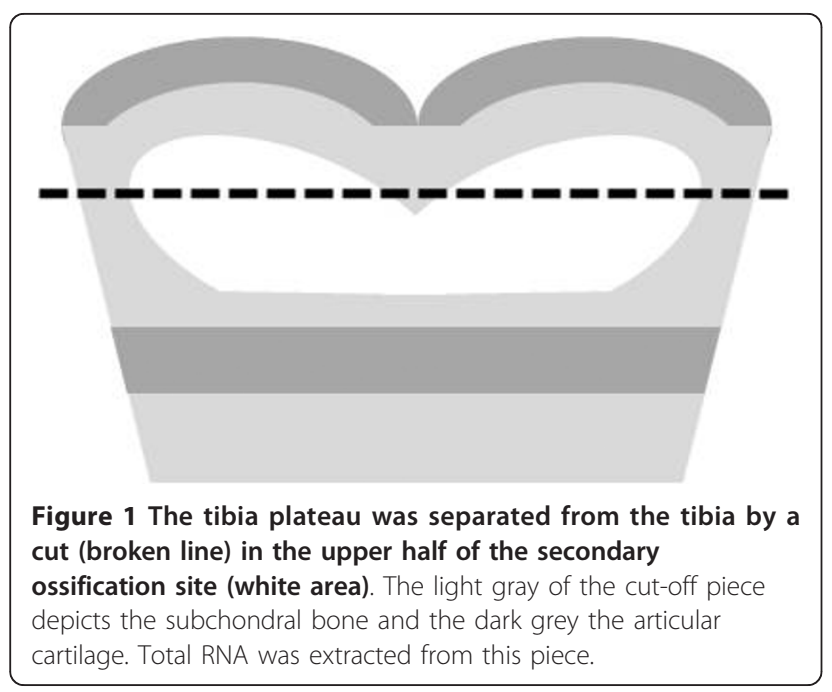

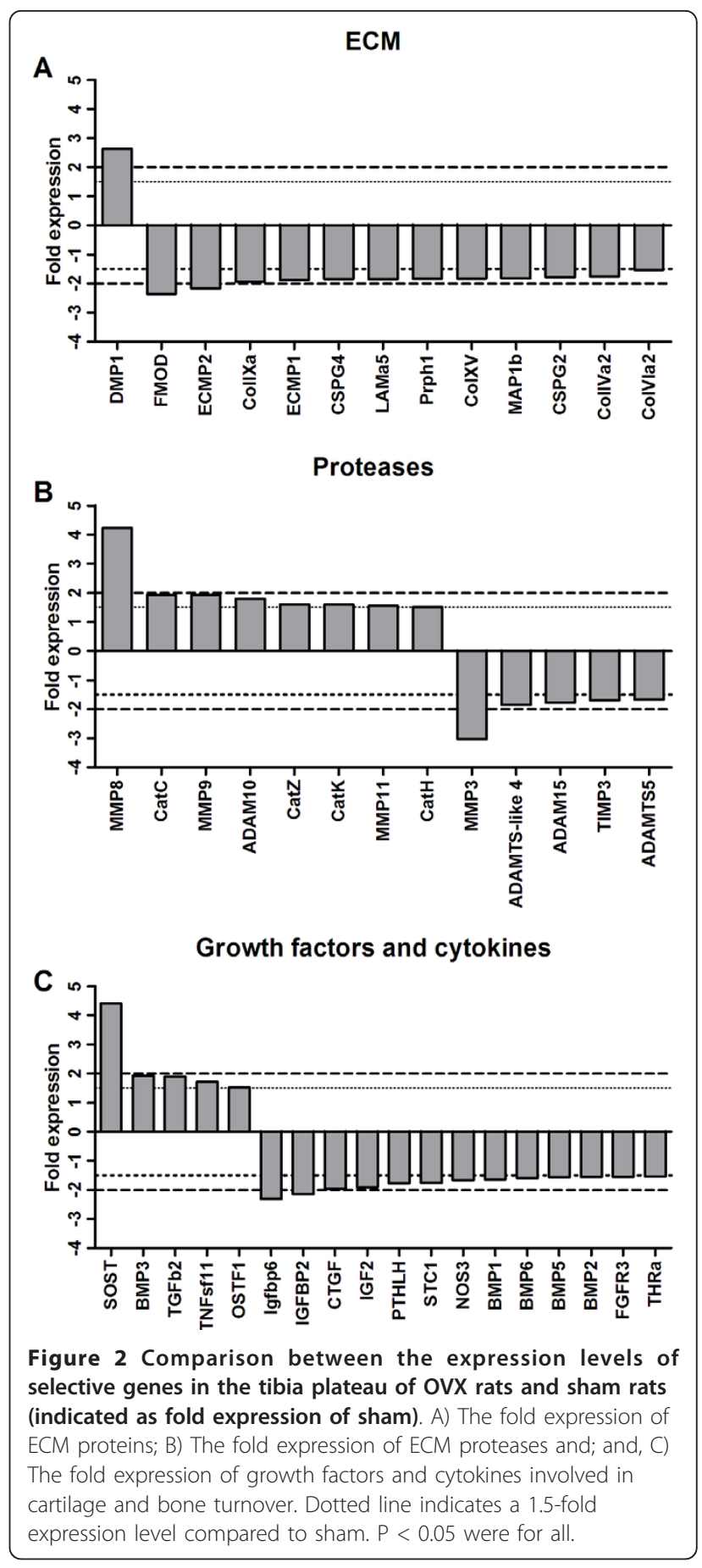

the tissue. Several collagens $(\mathrm{Col})$ were down-regulated; type IVa2, VIa2, IXa and XV. Type VI and IX are some of the minor collagens of the mature cartilage that are known to interact with other matrix components, such as hyaluronan, heperan sulfate and Chondroitin sulfate proteoglycans. Type XV collagen is member of the FACIT collagen family (fibril-associated collagens with interrupted helices) and mainly expressed by fibroblastic 
cells [12]. Type IV collagen, is a collagen of the basal lamina and widely distributed throughout the body, like many other collagens it is involved in tissue stabilization and flexibility [12]. Two chondroitin sulfate proglycans were also down-regulated in the joint tissue of OVX rats: CSPG2 (Versican) and 4 (NG2) (Figure 2A). Both have been shown to be involved in the regulations of bone development, chondrogenesis and angiogenesis, but only present in low amounts in mature cartilage and bone [13]. Laminin alpha 5 (LAMa5), a subunit of laminin 5 , which is observed in the basement membrane. It has been shown to promote bone growth and regeneration through cell adhesion and motility [14].

\section{Expression of ECM degrading proteases in response to estrogen deficiency}

Eight ECM degrading proteases were up-regulated in the joints of the OVX rats (Figure 2B). Metalloproteinase (MMP) 8 (collagenase-2), 9 (gelatinase B), 11 (stromelysin-3) and 16 (MT3-MMP) are collagen and proteoglycan degrading protease, which have been implicated as drivers of joint degradation [15]. Cathepsins (Cat) C, Z, $\mathrm{K}$ and $\mathrm{H}$, which are all cysteine proteases, have been shown to play vital role the regulation of tissue turnover. CatC is a dipeptidyl aminopeptidase, which is involved in the activation of serine proteases such as CatG and elastase [16]. CatZ is also a dipeptidyl peptidase, but its function in cartilage and bone turnover is unclear. CatK is mainly expressed by osteoclast and are responsible for the release of the bone turnover marker CTX-I. It has been found to be elevated in OVX animals and post-menopausal women. $\mathrm{CatH}$ cleaves osteocalcin in bone and might therefore be an important protease in bone turnover [17]. The A Disintegrin And Metallopeptidase 10 (ADAM10) is expressed by chondrocyte in bone development and in OA chondrocytes. It is therefore believed to be involved in the remodeling of cartilage [18].

Six proteases were down-regulated; MMP3 and the aggrecanases ADAMTS-like 4, ADAM15 and ADAMTS5, as well as bone morphogenic protein (BMP) 1 and tissue inhibitor metalloproteinase 3 (TIMP3) (Figure 2B). MMP3 can degrade fibrillar collagens such as type II and I collagen [19]. MMP3 has been shown to be elevated in inflammatory diseases, such as rheumatoid arthritis; however it is mainly expressed by the connective tissue like the synovial membrane and to a lesser degree by bone and cartilage. ADAM15 has been shown to cleave ECM components such as gelatin and type IV collagen. It is has been found to be upregrulated in OA chondrocytes [20]. ADAMTS-like 4 and ADAMTS5 (ADAM with thrombin mortif) are known for their capability to degrade proteoglycans such as aggrecan and versican [21]. It intriguing that ADAMTS5 is down-regulated, since previous studies have indicated it to be a primary protease in OA cartilage degradation [22]. In contrast, others have shown ADAMTS' are involved in both degradation and remodeling of the tissue $[23,24]$. The endogenous MMP and aggrecanase inhibitor TIMP3 was also down-regulated. TIMP3 is believed to be an important regulator of cartilage and bone turnover, through its inhibition of MMPs and aggrecanases [21]. In contrast to other BMPs, BMP1 does not belong to the TGF- $\beta$ family of proteins. It is a metalloproteinase that acts on procollagen I, II, and III and has been implicated in cartilage development [25].

\section{Expression of cytokines and growth factors in response to estrogen deficiency}

Next we searched for factors directly involved in bone and/or cartilage regulation. We found that five genes were significantly up-regulated and 11 were significantly down-regulated in the OVX animals (Figure 2C). Sclerostin (SOST) was markedly over-expressed in the OVX rats. It is expressed by osteoclast and is a negative regulator of bone formation through activation of the Wnt pathway [26]. BMP2 through BMP7 belong to the transforming growth factor beta superfamily of proteins. Whereas BMP6, -5 and -2 were down-regulated, BMP3 was up-regulated. BMP3 is known to induce bone formation and cartilage development and to have antagonistic effect on other BMPs [27]. Transforming growth factors beta-2 (TGFb2) expression was also elevated. TGFb2 is a multi-factorial regulator of cellular growth in developing systems and in repair of adult bone and cartilage [28]. TNFsf11, also known as RANKL, is a natural and necessary surface-bound molecule that activates osteoclasts, cells involved in bone resorption. Overproduction of RANKL is implicated in a variety of degenerative bone diseases, such as arthritis [29]. Osteoclast stimulating factor 1 (OSTF1) expression was also increased in the OVX rats' joints (Figure 2C). It is known to increase osteoclast formation and bone resorption through signal transduction, however only little is known about the family member of osteoblast stimulation factors [30]. Insulin-like growth factor 2 (IGF2), IGF binding protein 2 (IGFBP) and IGFBP6 were all down-regulated in the OVX rats. IGF2 is known to bind both to IGFBP2 and IGFBP6, and its effect on bone cells is thereby prevented (e.g. osteoblast proliferation) [31]. Connective tissue growth factor (CTGF) is a potent stimulator for proliferation and differentiation of osteoblast and chondrocytes through regulation of cellular associated genes [32]. The tightly related proteins Fibroblast growth factor receptor 3 (FGFR3) and the parathyroid hormone-like hormone (PTHLH), which were both down-regulated, play central roles in the physiological regulation of bone formation, 
by promoting recruitment and survival of osteoblasts, and probably plays a role in the physiological regulation of bone resorption, by enhancing osteoclast formation. Signaling by FGFR3 and PTHLH coordinates cartilage and bone development. PTHLH is also an essential physiological regulator of adult bone mass [33]. The calcitropic hormone Stanniocalcin 1 (STC1) was downregulated in the OVX rats. It is involved in the regulation of longitudinal bone growth [34]. Nitric oxide synthase 3 (NOS3, eNOS) was also down-regulated. It has been shown that estrogen induces NOS3 expression in endothelial cells and osteoblasts, raising the possibility that NO derived from the NOS3 pathway plays a role in mediating the effects of sex hormones on bone [35]. BMP2, -5 and -6 , which were down-regulated in the OVX rats (Figure 2C), all induces cartilage and bone formation and play role in joint integrity [27].

\section{Discussion and Conclusion}

We were interested in investigating the effect of ovariectomy (i.e. estrogen deficiency) on the joint. Here we use a rodent model that in previous study has been shown to develop bone and cartilage changes, resembling pathological features of OA. We have made a guided search on ECM molecules, matrix proteases and cytokines/growth factors that have previously been directly implicated in bone and cartilage turnover. Although the search was made electronically, it is still somewhat subjective, because the database keywords may not be ubiquitous and fulfilling. Nevertheless we found several differentially express genes, which are known to play a role in development of joint degenerative disease such as OA.

Animal studies have shown that by inhibiting osteoclast activity, the histo-pathological score (cf. Mankin) could be markedly reduced, suggesting that osteoclasts play a central role in OA and cartilage breakdown. Also bone formation cells, osteoblast, seem to play an important role in OA: osteoblasts isolated from subchondral bone of OA hips demonstrate an altered and more active phenotype. Furthermore, IGF-1 (a bone promoting factor as well as several growth factors (e.g. BMPs, TGF and FGF) has been shown to be elevated in OAsubchondral explant cultures.

In conclusion, we found that extracellular matrix proteins, proteases and paracrine factors known to be involved in joint degenerative diseases were either down- or up-regulated in response to estrogen-depletion. As a whole, the data support current knowledge; however there are some discrepancies, which stresses that more investigation is needed to fully understand the pathogenesis of post-menopausal OA.

\section{Acknowledgements}

We would like acknowledge the Danish research fund for an unrestricted grant.

\section{Author details}

'Cartilage biology and biomarkers, Nordic Bioscience, Herlev, Denmark. ${ }^{2}$ Novartis Institutes for BioMedical Research, Novartis Pharma AG, Basel, Switzerland

\section{Authors' contributions}

Conception and design: RHN, MAK, AB. Analysis and interpretation of the data: ACBJ. Drafting of the article: ACBJ. Critical revision of the article for important intellectual content, MAK, MA, NH, FS and ML. Final approval of the article: MAK. Provision of study materials or patients: N/A. Statistical expertise: AB. Obtaining of funding: MAK. Administrative, technical, or logistic support: N/A. Collection and assembly of data: ACBJ. All authors have approved this final version of the manuscript.

\section{Competing interests}

The authors declare that they have no competing interests.

Received: 11 November 2010 Accepted: 15 March 2011

Published: 15 March 2011

\section{References}

1. Nevitt MC, Felson DT: Sex hormones and the risk of osteoarthritis in women: epidemiological evidence. Ann Rheum Dis 1996, 55:673-6.

2. Felson DT, Nevitt MC: The effect of estrogen on osteoarthritis. Curr Opinion in Rheumatology 1998, 10:269-72.

3. Kalu DN: The ovariectomized rat model of postmenopausal bone loss. Bone Miner 1991, 15:175-91.

4. Richmond RS, Carlson CS, Register TC, Shanker G, Loeser RF: Functional estrogen receptors in adult articular cartilage. Estrogen replacement therapy increases chondrocyte synthesis of proteoglycans and insulinlike growth factor binding protein 2. Arthritis Rheum 2000, 43:2081-90.

5. Turner AS, Athanasiou KA, Zhu C-F, Alvis MR, Bryant HU: Biochemical effects of estrogen on articular cartilage in ovariectomized sheep. Osteoarthritis and Cartilage 1997, 5:63-9.

6. Hoegh-Andersen $\mathrm{P}$, Tanko L, Andersen T, et al: Ovariectomized rats as a model of postmenopausal osteoarthritis: validation and application. Arthritis Res Ther 2004, 6:R169-R180.

7. Bay-Jensen AC, Charni-Ben Tabassi N, Sondergaard LV, et al: The response to estrogen deprivation of the cartilage collagen degradation marker, CTX-II, is unique compared to other markers of collagen turnover. Arthritis Res Ther 2009, 11:R9.

8. Sniekers $\mathrm{YH}$, Weinans $\mathrm{H}$, Bierma-Zeinstra SM, van Leeuwen JP, van Osch GJ: Animal models for osteoarthritis: the effect of ovariectomy and estrogen treatment - a systematic approach. Osteoarthritis Cartilage 2008, 16:533-41.

9. Brachat A, Pierrat B, Brungger A, Heim J: Comparative microarray analysis of gene expression during apoptosis-induction by growth factor deprivation or protein kinase C inhibition. Oncogene 2000, 19:5073-82.

10. George A, Gui J, Jenkins NA, Gilbert DJ, Copeland NG, Veis A: In situ localization and chromosomal mapping of the AG1 (Dmp1) gene. $J$ Histochem Cytochem 1994, 42:1527-31.

11. Heinegard D, Oldberg A: Structure and biology of cartilage and bone matrix noncollagenous macromolecules. FASEB J 1989, 3:2042-51.

12. Gelse K, Poschl E, Aigner T: Collagens-structure, function, and biosynthesis. Advanced Drug Delivery Reviews 2003, 55:1531-46.

13. Matsumoto K, Kamiya N, Suwan K, et al: Identification and characterization of versican/PG-M aggregates in cartilage. J Biol Chem 2006, 281:18257-63.

14. Hashimoto J, Kariya Y, Miyazaki K: Regulation of proliferation and chondrogenic differentiation of human mesenchymal stem cells by laminin-5 (laminin-332). Stem Cells 2006, 24:2346-54.

15. Krane SM, Inada M: Matrix metalloproteinases and bone. Bone 2008, 43:7-18.

16. Adkison AM, Raptis SZ, Kelley DG, Pham CT: Dipeptidyl peptidase I activates neutrophil-derived serine proteases and regulates the development of acute experimental arthritis. J Clin Invest 2002, 109:363-71. 
17. Baumgrass R, Williamson MK, Price PA: Identification of peptide fragments generated by digestion of bovine and human osteocalcin with the lysosomal proteinases cathepsin B, D, L, H, and S. J Bone Miner Res 1997, 12:447-55.

18. Chubinskaya S, Mikhail R, Deutsch A, Tindal MH: ADAM-10 protein is present in human articular cartilage primarily in the membrane-bound form and is upregulated in osteoarthritis and in response to IL-1alpha in bovine nasal cartilage. J Histochem Cytochem 2001, 49:1165-76.

19. Rengel Y, Ospelt C, Gay S: Proteinases in the joint: clinical relevance of proteinases in joint destruction. Arthritis Research \& Therapy 2007, 9:221.

20. Bohm BB, Schirner A, Burkhardt h: adam15 modulates outside-in signaling in chondrocyte-matrix interactions. $j$ Cell Mol Med 2008.

21. Huang K, Wu LD: Aggrecanase and aggrecan degradation in osteoarthritis: a review. J Int Med Res 2008, 36:1149-60.

22. Glasson SS, Askew R, Sheppard B, et al: Deletion of active ADAMTS5 prevents cartilage degradation in a murine model of osteoarthritis. Nature 2005, 434:644-8.

23. Li J, Anemaet W, Diaz MA, et al: Knockout of ADAMTS5 does not eliminate cartilage aggrecanase activity but abrogates joint fibrosis and promotes cartilage aggrecan deposition in murine osteoarthritis models. J Orthop Res 2010.

24. Bay-Jensen AC, Hoegh-Madsen S, Dam E, et al: Which elements are involved in reversible and irreversible cartilage degradation in osteoarthritis? Rheumatol Int 2009.

25. Ge G, Greenspan DS: BMP1 controls TGFbeta1 activation via cleavage of latent TGFbeta-binding protein. J Cell Biol 2006, 175:111-20.

26. Kubota T, Michigami T, Ozono K: Wnt signaling in bone metabolism. Bone Miner Metab 2009, 27:265-71.

27. Bahamonde ME, Lyons KM: BMP3: to be or not to be a BMP. J Bone Joint Surg Am 83-A 2001, Suppl 1:S56-S62.

28. Jenniskens $Y M$, Koevoet $W$, de Bart AC, et al: Biochemical and functional modulation of the cartilage collagen network by IGF1, TGFbeta2 and FGF2. Osteoarthritis Cartilage 2006, 14:1136-46.

29. McClung MR: Inhibition of RANKL as a Treatment for Osteoporosis: Preclinical and Early Clinical Studies. Curr Osteoporos Rep 2006, 4:28-33.

30. Tong S, Zhou H, Gao Y, et al: Crystal structure of human osteoclast stimulating factor. Proteins 2009, 75:245-51.

31. Conover CA: Insulin-like growth factor-binding proteins and bone metabolism. Am J Physiol Endocrinol Metab 2008, 294:E10-E14.

32. Nishida T, Kawaki H, Baxter RM, Deyoung RA, Takigawa M, Lyons KM: CCN2 (Connective Tissue Growth Factor) is essential for extracellular matrix production and integrin signaling in chondrocytes. J Cell Commun Signal 2007, 1:45-58.

33. Adams SL, Cohen AJ, Lassova L: Integration of signaling pathways regulating chondrocyte differentiation during endochondral bone formation. J Cell Physiol 2007, 213:635-41.

34. Wagner GF, Dimattia GE: The stanniocalcin family of proteins. J Exp Zoolog A Comp Exp Biol 2006, 305:769-80.

35. van't Hof RJ, Ralston SH: Nitric oxide and bone. Immunology 2001, 103:255-61.

doi:10.1186/1756-0500-4-63

Cite this article as: Bay-Jensen et al:: A microarray analysis of full depth knee cartilage of ovariectomized rats. BMC Research Notes 2011 4:63.

\section{Submit your next manuscript to BioMed Central and take full advantage of:}

- Convenient online submission

- Thorough peer review

- No space constraints or color figure charges

- Immediate publication on acceptance

- Inclusion in PubMed, CAS, Scopus and Google Scholar

- Research which is freely available for redistribution

Submit your manuscript at www.biomedcentral.com/submit 